\title{
Interação Coletiva Inteligente para Suporte à Vida Independente de Idosos
}

\author{
Ana Cristina B Garcia \\ Universidade Federal do Estado \\ do Rio de Janeiro \\ Rio de Janeiro, Brasil \\ cristina.bicharra@uniriotec.br
}

\author{
Adriana S Vivacqua \\ Universidade Federal do \\ Rio de Janeiro \\ Rio de Janeiro, Brasil \\ avivacqua@dcc.ufrj.br
}

\begin{abstract}
Este artigo descreve as linhas de pesquisa do grupo SCAI (Sistemas Colaborativos para Apoio a Idosos) em sistemas colaborativos no domínio de vida assistida usando dispositivos móveis. Enfatizamos questões estruturais, sociais e tecnológicas da interação entre dispositivos tecnológicos e os vários atores no cuidado ao idoso. A equipe atual de pesquisa é composta por 4 professores e 7 alunos.
\end{abstract}

\section{Author Keywords}

Acessibilidade; Envelhecimento; Emergência; Crowdsourcing; Computação em Multidões.

\section{ACM Classification Keywords}

H.5.m. Information interfaces and presentation (e.g., HCI): Miscellaneous.

\section{INTRODUÇÃO}

Dados da ONU mostram que a população mundial está envelhecendo rapidamente. As projeções são de que pessoas com 60 anos ou mais serão mais numerosas que crianças $(0$ a 9 anos) até 2030, e superarão os adolescentes e jovens (com idade entre 10 e 24 anos) até 2050. O envelhecimento leva a degradação sensorial gradual, o que aumenta a dependência de outras pessoas. A medida que a população mundial envelhece, aumenta a necessidade de políticas, sistemas e tecnologias para apoiar o envelhecimento saudável.

Envelhecer é um processo gradual que não se sabe ao certo quando se inicia, e nem sempre está claro quando se precisa de ajuda. A tecnologia pode ajudar nesse período de transição, em especial as instaladas em dispositivos móveis, por serem artefatos comuns do dia a dia, já adotados por muitos idosos. Além destes existem ainda dispositivos vestíveis ou mesmo aqueles sem contato direto com o idoso.

Manter-se independente e com segurança é um desafio para os idosos e uma fonte de preocupação para suas famílias.

\footnotetext{
Permission to make digital or hard copies of all or part of this work for personal or classroom use is granted without fee provided that copies are not made or distributed for profit or commercial advantage and that copies bear this notice and the full citation on the first page. Copyrights for components of this work owned by others than the author(s) must be honored. Abstracting with credit is permitted. To copy otherwise, or republish, to post on servers or to redistribute to lists, requires prior specific permission and/or a fee. Copyright 2018 SBC.
}

IHC 2018, Anais Estendidos do XVII Simpósio Brasileiro sobre Fatores Humanos em Sistemas Computacionais Outubro 22-26, 2018, Belém, Brasil

Fórum de Integração e Discussão sobre Interação Humano-Computador e Sistemas Colaborativos (FID IHC-SC)
Durante atividades rotineiras, até mesmo idosos saudáveis podem se perder, ficar expostos a calor excessivo ou a baixas temperaturas, cair, ficar confusos ou experimentar eventos relacionados a saúde. Em um ambiente amigável, onde as pessoas conhecem e cuidam umas das outras, o bairro ajudaria os idosos em risco, seja chamando um cuidador, membro da família ou até mesmo uma ambulância.

Tecnologias de "Ambient Assisted Living" (AAL) (Ambientes Assistivos) prolongam a capacidade das pessoas de se manterem ativas e independentes mesmo com o avançar da idade. No entanto, a maioria das pesquisas em AAL enfoca ambientes internos (residenciais), usando informações de sensores no ambiente e na pessoa. Porém, muitas atividades são realizadas fora do ambiente doméstico, expandindo a necessidade de monitoramento e trazendo outras preocupações de segurança. Além disso, muitas soluções AAL exigem que os idosos realizem ações (pressionar um botão, fazer uma ligação, etc.), o que, dependendo da situação, pode não ser possível. Estamos explorando o uso de técnicas AAL em ambientes externos para aumentar a independência e segurança de idosos, evitando a necessidade de interação com a tecnologia. Pesquisas atuais em monitoramento externo utilizam exclusivamente dados de sensores.

É neste cenário que desenvolvemos a pesquisa do grupo: o objetivo é aumentar a independência do idoso, provendo maior segurança em ambientes internos e externos. A seguir, descrevemos nossa, abordagem, a "Vizinhança Segura" (VS), seguida da explicação das questões de estudo e da apresentação da equipe de pesquisa. Concluímos com um mapeamento entre as questões do nosso estudo em sistemas colaborativos, com questões da área de IHC.

\section{ABORDAGEM: VIZINHANÇA SEGURA}

A VS, adota abordagens de inteligência artificial para monitoramento de atividades e inclui uma camada de computação humana fornecida pela terceirização de tarefas a pessoas que se encontram fisicamente próximas ao idoso, revisando inferências computacionais sobre dados contextuais e de sensores.

Na VS, um sistema implementado em um dispositivo móvel é responsável por detectar possíveis situações de emergência que venham a ocorrer com o idoso, utilizando técnicas de fusão e análise de dados para processar dados obtidos a partir 
de sensores do smartphone, inferindo se o idoso está em situação de risco (queda, exposição ao calor ou frio excessivos ou desorientado.)

Para reduzir a incerteza, essas inferências são enviadas para as pessoas que estão nas proximidades, para que estas confirmem o diagnóstico do sistema, e prestem auxílio ao idoso caso necessário. Nesse momento, uma pessoa na rua (ex: jornaleiro, porteiro, lojista, etc.) que tenha visto ou tido contato com o idoso pode rapidamente confirmar que ele/a esteve em determinado local e se está em condição segura. Esses indivíduos formam uma rede de segurança para o idoso, que pode sair de casa desacompanhado pois a comunidade estará atenta as suas necessidades.

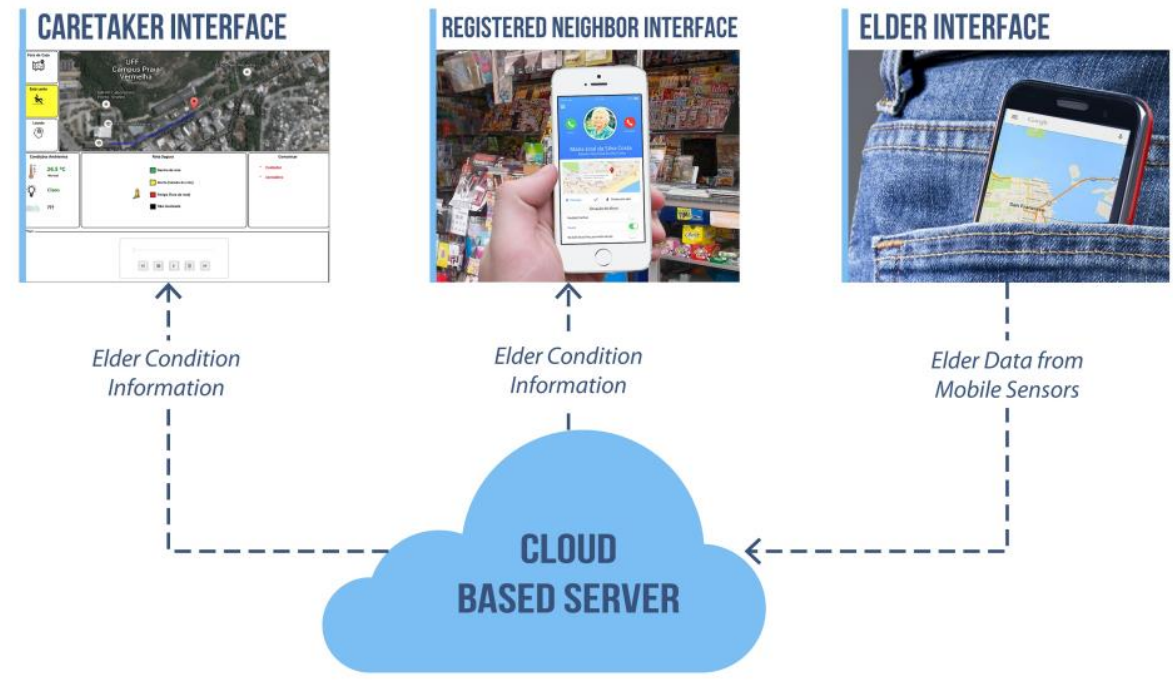

Figura 1. Esquema do Projeto Vizinhança Segura.

Além dos aplicativos para o idoso e para os "vizinhos", o sistema conta com uma interface em back-end que permite a um responsável (familiar e/ou cuidador) monitorar a situação do idoso, e receber notificações em caso de urgência. Dados são armazenados em um repositório centralizado, que é acessado por todos os sistemas pertinentes. O esquema proposto é ilustrado na Figura 1.

Esta solução levanta questionamentos em relação a privacidade e segurança do idoso (considerando que este é presa fácil, o envio de um alerta de que uma pessoa está passando mal pode acabar atraindo elementos mal intencionados.) Além de questões de interação (deve ser o mais simples possível, sob risco dos vizinhos não responderem aos alertas/perguntas) e de incentivos à participação, discutidas em maiores detalhes a seguir.

\section{QUESTÕES DE PESQUISA}

O contexto da Vizinhança Segura envolve questões de pesquisa de natureza diferentes: sociais, provenientes da interação entre os diversos atores considerados na abordagem, culturais e éticas provenientes da intrusividade do monitoramento direto ou indireto, e tecnológicas, provenientes da implementação da abordagem.

\section{Questões Sociais}

Estamos em fase de estudo exploratório para entender melhor as oportunidades que as tecnologias de mídias sociais oferecem para aumentar a inclusão do idosos. Além disso, como há diversos atores diferentes, com demandas e permissões de acesso à informação diferentes, estamos interessados em entender como esses atores podem otimizar suas tarefas de grupo para dar assistência ao idoso.

Inclusão:

As mídias sociais são importante ferramentas para o engajamento e socialização de idosos. WhatsApp, Facebook, Instagram... são aliados para acompanhar a vida dos filhos e netos. A inclusão previne a depressão em idosos, porém há estudos que evidenciam uma dificuldade dos idosos de acompanhar a comunicação nesses grupos. A barreira de entrada ainda é grande. Estamos focando nossos estudos no WhatsApp [3]. Já fizemos um estudo qualitativo inicial com entrevistas e questionários que nos trouxe constatações interessantes, em via de publicação. Estamos incluindo um aluno para participar de um estudo sobre os textos no WhatsApp usando etnografia. Especificamente para a abordagem de VS, estamos interessados em usar tais mídias como meio de naturalizar e humanizar o monitoramento.

\section{Interação diversificada:}

Existem vários participantes atuando na abordagem de Vizinhança Segura: o idoso, parentes, cuidadores, equipe médica, e os vizinhos. A comunicação direta e indireta entre esses atores; a colaboração da coletividade; e o acompanhamento individual e coletivo do monitoramento são algumas das questões da nossa investigação.

\section{Desfocagem:}

As questões de estudo envolvem entender como a tecnologia assistiva pode auxiliar adultos nas transições entre as três primeiras fases da velhice: pré-aposentadoria; vida 
independente como aposentado; vida dependente precoce (com limitações crescentes). Planejamos estudos etnográficos para entender melhor os requisitos para o design de tecnologias assistivas desta natureza.

\section{Suporte à Adaptação:}

Acompanhar a pessoa à medida que ela se torna mais seletiva sobre o que faz, mas também ajudando-a a escolher seu caminho, reconhecendo e apoiando o trabalho de gerenciar o envelhecimento, bem como o processo de envelhecimento. Esse será um estudo longitudinal que ainda se encontra em fase de planejamento.

\section{Questões Culturais e Éticas}

Segurança e Privacidade do idoso:

Estamos interessados em entender os requisitos de segurança das informações gerais, tanto do idoso quanto dos participantes da vizinhança, bem como a segurança dos registros médicos do idoso. Contudo, o que orienta nossa pesquisa é a dicotomia entre prover segurança para a independência do idoso nas atividades através do monitoramento e violar sua privacidade ao revelar seu cotidiano a outros, mesmo seus parentes.

Tons de privacidade:

Nos interessa ainda investigar o papel do anonimato dos participantes em contraste com a reputação da informação, os incentivos para participação e as consequências pela participação, e como essas questões podem fomentar ou destruir a proposta da VS.

\section{Questões Tecnológicas}

A abordagem da VS envolve muitas questões tecnológicas de hardware e software que precisam ser resolvidas para viabilizar sua implementação. A seguir, listamos algumas nas quais estaremos trabalhando nos próximos três anos.

\section{Interação estendida:}

Essas questões envolvem a formação, interação e manutenção de uma rede social e de assistência médica de apoio ao idoso. Há três focos de pesquisa: segurança das informações, fusão de dados e usabilidade na interação com idosos. Ainda estamos em fase de estudo exploratório com um aluno de mestrado criando o ambiente computacional para o estudo. Já temos resultados da fusão de dados [1].

\section{Monitoramento das atividades:}

Questões incluem a determinação dos artefatos para coleta de dados, análise dos dados para determinação das atividades, diagnóstico do grau de risco de anomalias com idoso. O foco está na acurácia dos resultados e no tempo de resposta. Não adianta diagnosticar uma situação de emergência depois que o idoso morreu. Técnicas estatísticas e de aprendizado de máquina estão sendo estudadas por um aluno de doutorado [2].

\section{Criação e Manutenção de Vizinhança Segura:}

Questões envolvem a criação de um modelo de incentivo que promova a participação dos "vizinhos" como "sensores vivos". Também há questões envolvendo a reputação das informações coletadas e a agregação das mesmas [4].

\section{CONCLUSÕES}

A saúde dos idosos é uma questão importante e que merece mais atenção. Questões de acessibilidade, frequentemente estudadas em IHC, são muito pertinentes neste projeto, bem como o projeto de usabilidade voltado para simplicidade e mecanismos de incentivo que levem os vizinhos a se engajar no sistema. Qualquer abordagem que se valha da coletividade para auxiliar o idoso e que para tal deve distribuir informações sobre eles levanta questões relativas à privacidade e acesso ao sistema. Métodos de pesquisa em IHC são apropriados para este projeto, no qual vemos bastante interseção entre as áreas de IHC e CSCW.

\section{PESQUISADORES SCAI}

A equipe SCAI envolvida atualmente no projeto consiste de 11 pesquisadores, listados a seguir.

- Ana Cristina Bicharra Garcia, professora, UNIRIO

- Simone Bacellar, professora, UNIRIO

- Adriana S Vivacqua, professora, UFRJ

- Leandro Fernandes, professor, UFF

- Igor Natal, aluno doutorado, UFF

- Julliano Pintas, aluno doutorado, UFF

- Wagner Silva, aluno doutorado, UNIRIO

- Orete Nascimento, aluno doutorado, UNIRIO

- Luiz Ribeiro, aluno doutorado, UNIRIO

- Lívia Carolina Gouvea, aluna mestrado, UNIRIO

- Isabella Lima, aluna mestrado, UFRJ

\section{REFERENCIAS}

1. Javier J Alemán, Nayat Sánchez-Pi, Luis Marti, José M Molina, Ana Cristina B Garcia. 2016. A Data Fusion Model for Ambient Assisted Living. In International Conference on Practical Applications of Agents and Multi-Agent Systems. Springer, 301-312.

2. Igor P Natal, Rogerio A C Cordeiro, Ana Cristina B Garcia. 2017. Activity Recognition Model Based on GPS Data, Points of Interest and User Profile. In International Symposium on Methodologies for Intelligent Systems. Springer, 358-367.

3. Ana Cristina B. Garcia, A. Vivacqua. 2019. A Data Fusion Model for Ambient Assisted Living. In 52nd Hawaii International Conference on Systems Science. Under review.

4. Ana Cristina B Garcia, Adriana S Vivacqua, Nayat Sánchez-Pi, Luis Martí, José M Molina. 2017. Crowd-Based Ambient Assisted Living to Monitor the Elderly's Health Outdoors. IEEE Software 34, 6 (2017), 53-57. 